\title{
Expression and Methylation Pattern of hsa-miR-34 Family in Sperm Samples of Infertile Men
}

\author{
Abdolmabood Momeni $^{1,2} \cdot$ Reza Najafipour $^{1} \cdot$ Ahmad Hamta $^{2} \cdot$ Suzan Jahani ${ }^{3} \cdot$ Sahar Moghbelinejad $^{1}$
}

Received: 13 January 2019 / Accepted: 1 April 2019

(C) Society for Reproductive Investigation 2019

\begin{abstract}
Production of high-quality spermatozoa is necessary for male fertility. In this regard, post-mitotic stage in spermatogenesis is very important which posttranscriptional microRNAs (miRNAs) playing a key role at this stage. In this research, we evaluated the expression and methylation of hsa-miR-34 family in sperm samples of infertile men. We recruited 102 infertile men (asthenozoospermia, teratozoospermia, asthenoteratozoospermia, and oligoasthenoteratozoospermia) and 52 fertile men as control. The expression of $h s a-m i R-34 a, b, c$ was determined by quantitative real-time PCR (qRT-PCR) technique. Methylation of $h s a-m i R-$ $34 b, c$ promoter was evaluated by methylation-specific PCR (MS-PCR) method. Our data indicated under-expression of three miRNAs (hsa-miR-34a,b,c) in the sperm samples of infertile men in compared to their fertile counterparts. The highest rate of expression reduction was observed in $h s a-m i R-34 c-5 p$ and in oligoasthenoteratospermic patients $(P=0.011, \mathrm{~F}=4.01)$. The results revealed that the frequency of methylation of the promoter region of $h s a-m i R-34 b, c$ in infertile men was higher than that of fertile men $(82.4 \%$ versus $23.3 \%$ ), and the highest frequency of methylation was observed in patients with asthenoteratospermia (92.9\%) and oligoasthenoteratospermia (93.8\%). In conclusion, our results indicated lower expression of $h s a-m i R-34 a, b, c$ and hypermethylation of $h s a-m i R-34 b, c$ promoter in sperm samples of infertile men. Aberrant under-expression of these miRNAs could be duo to the hypermethylation of the promoter region and indicative of a defect in spermatogenesis.
\end{abstract}

Keywords Male infertility $\cdot h s a-m i R-34$ family $\cdot$ Epigenetic

\section{Introduction}

Infertility usually afflicts $10-15 \%$ of couples [1] and $50 \%$ of infertility is related to male factor. Meanwhile, $60-75 \%$ of infertility in men are idiopathic, and the precise mechanism for its occurrence is unclear [1]. Many genetic factors interfere with male infertility, among which Yq microdeletion and

Electronic supplementary material The online version of this article (https://doi.org/10.1007/s43032-019-00025-4) contains supplementary material, which is available to authorized users.

Sahar Moghbelinejad

smoghbelinejad@qums.ac.ir

1 Cellular and Molecular Research Centre, Qazvin University of Medical Sciences, P.O. Box: 341197-5981, Qazvin, Iran

2 Biology Department, School of Basic Science Arak University, Arak, Iran

3 Telemedicine Infertility Treatment Center, ACECR branch of Qazvin, Qazvin, Iran chromosomal aberration are the main causes of impaired spermatogenesis and male infertility [2].

On the other hand, the role of microRNAs (miRNAs) in male infertility has been highlighted these days. miRNAs are small (18-25 nt) RNAs categorized as noncoding RNAs, and their function is to regulate gene expression [3]. They have close to $90 \%$ sequence homology among the human, mouse, and rat. [4] miRNAs control the gene expression in two ways: by suppressing translation/transcription (RNAi) [5] or by activating transcription (RNAa). [6] The results of computational analyses have indicated the presence of 50,000 miRNAs in mammalian cells, where each miRNA regulates a great deal of mRNA expression [7].

In this way, they control the expression of many genes and have a vital role in important cellular processes such as development, differentiation, apoptosis, and proliferation processes in various species $[3,5,7,8]$. Studies have suggested that miRNAs are involved in different stages of male germ cell development, where some of their expression is limited to certain types of male germ cells, while the others are expressed in different cell types of the testes [9-11]. 